\title{
A Kind of Exchange System for Product Conceptual Design Based On the Enterprise Network Community
}

\author{
Dianting Liu ${ }^{1, a}$, Quanyong Zhang ${ }^{2, b}$ \\ ${ }^{1}$ College of Mechanical and Control Engineering, Guilin University of Technology Guangxi Guilin \\ 541004, China \\ ${ }^{2}$ College of information science and engineering, Guilin University of Technology Guangxi Guilin \\ 541004, China

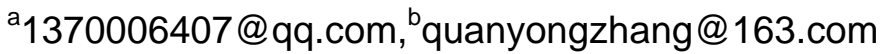

Keywords: Product conceptual design; Enterprise network community; Interactive communication

\begin{abstract}
On the basis of the analysis on product conceptual design, four problems have been found. In order to solve the problems, the article puts forward a kind of communication system for the product conceptual design based on enterprise network community. In the system the Internet users are divided into lead users and ordinary users, having the function of self-learning, and exchanging information in two-layer. From the effect of the system implementation, the four problems have been improved to a certain extent. After the practical application for several months, this system is good in performance and proper in operation, and improves the generating speed and efficiency of product conceptual design scheme.
\end{abstract}

\section{Introduction}

Conceptual design is the core stage of product innovation [1]. At the same time, the product conceptual design is innovative thinking and innovative ideas to the most effective and most useful ways, according to the research, product conceptual design accounted for about $5 \%$ of the total cost of development, but decided $70 \%-80 \%$ of the products performance, value and efficiency [2]. Therefore, the concept of a product design is good or bad almost determines the success or failure of a product. Although now the academic study of product conceptual design invested a lot and has also made great achievements, but most of the research mainly by in modern theory and method, mainly rely on TRIZ (inventive problem solving theory) theory is used to solve the problem, for the analysis and definition of the problem by QFD, AD, AHP, KJ method, 5WHY analysis or will integrate these methods use. Now the research of the bottleneck lies in the analysis and definition of the problem that is the user demand acquisition, analysis and definition, for analysis and definition of problem now mainly rely on manual, such as QFD house of quality analysis, AHP to user needs hierarchical analysis, this is the problem one. Manual will extend the time, and thus have an impact on the concept of the entire product design cycle, now it is the information age, requirements at all stages of the development cycle as much as possible short because the market is constantly changing, this is the problem two. The product design participants are generally divided into five categories: user, concept designers, product managers, designers and process designers structure [3], the more the classification the more delay the development cycle, this is the problem three.The solutions by using TRIZ theory to solve the problem are TRIZ solutions, which is the general idea or a general solution, rather than the specific concept design, still need careful handling, this is the problem four. To solve the above four problems, the author combined with the network community [4] to its corresponding repair, and improve. 


\section{Related Work}

\subsection{Research status of product concept design}

In general, the concept design is the input for the design requirements, output for the best program of a system workflow [5]. The TRIZ theory is mainly used to assist designers to use analogical reasoning method to solve practical engineering problems, it does not give a specific process, so it is difficult to solution to complex multi-level innovation design problem tools. At present, TRIZ and QFD, TOC, AHP, AD, 5WHY, are combined to make up the deficiency of TRIZ [2]. Obviously, it is failed to link the concept design to the computer technology and network technology. Zhang Jianhui, Tan run Hua [1] 2013 proposed CAI driven product innovation idea generation principle, and to establish the CAI driven product conceptual design ideas for innovation model of generating, finally the product conceptual design and network technology to link up, however, the application of CAI software solution must innovation design problem and cannot directly get innovative solutions can only get product innovation TRIZ solution, which is the general idea or the general solution. Visible, product concept design and network technology integration has yet to be further studied.

\subsection{Research status of enterprise network community}

Network community is a network application platform which is a kind of information dissemination, communication, exchange and sharing. It is one of the network technology in the 21st century the most outstanding product, with some basic properties of natural: cross regional, time, mutual subjectivity, openness and anonymous. Due to these properties, one cannot subject to time, place, geographical position, professional background, status background influences the free exchange and sharing, the network community has been at breakneck speed into people's daily life. In addition, under the environment of network community information dissemination with three new characteristics [4]: (1) the information propagation direction reversible; (2) information sharing time to speed up; (3) information pointing to the target accurately. It can be seen that several basic properties of network community and the three characteristics of information dissemination can provide great convenience to solve the above problems. However, at present, the research of the network community mainly focus on the user classification, community discovery, information dissemination, social recommendation etc [6]. Obviously, the research of the network community is confined to the scope of its own domain, there is not much more than other fields and disciplines

\subsection{The exchange system for product conceptual design based on the enterprise network community is proposed}

From the above analysis, we know that product conceptual design based on the network community needs to be further in-depth study, study of network community has not extended to research in the field of product conceptual design. Network community can provide great convenience to solve the above problems. In addition, the professional development and design staff in the long-term work accumulated some valuable experience. Innovative thinking, divergent thinking is bound and very easy to the formation of the inertia of thinking, which in a certain extent hinder innovation in product conceptual design. At the same time, because of the characteristics of the interaction, communication and sharing of the network community, the divergent thinking is greatly stimulated. To sum up, the exchange system is proposed.

\section{A Kind Of Exchange System For Product Conceptual Design Based On The Enterprise Network Community}

The system used the Internet users as an alternative class of the past five categories users [3]. Therefore, the design process for each stage is no longer a specific participant to handle, and all network users can participate anytime, anywhere, so, to some extent, to optimize the problem three. According to lead user theory [7] of the network users into a leading users and the ordinary users, leading users are as early as a few months or even a few years ago to predict future demand for new 
products than ordinary users. Leading user can act as a leading role in Figure 1, the concept of the designer, product manager, structural designer, craft designer. Ordinary users can in problem analysis, solution evaluation processes play a role. Network users can from time, place, and time and space limit full exchange sharing system in the process of user communication automatically finished the analysis and definition, without the need of manual intervention, which to fix the problem one and two is helpful. In the system, the network users using CAD software embedded in a web page online design the product conceptual design solutions, by rendering the DWG file of the given their detailed design, which directly solves the problem four.

\subsection{System structure}

The system is shown in Figure 1. The structure of the system is clear and concise. Network users especially leading users through Internet, database knowledge and its knowledge accumulation retrieval, are free to construct a concept product or by embedding the CAD software drawing, or on the basis of existing products at any site to add their own design or put forward their own point of view, then put in the first layer exchange area for everyone to exchange, according to the product concept they can express views freely, it is conducive to understand and improvement of the product. After the first layer communication, users are already familiar with the product, then according to their own ideas and knowledge, submit their product conceptual design solution. At the same time, a set of conceptual design is presented. Users can see each design solution, can vote for them and a user can only vote once for a solution. The system select a better product solution according to the modeling formula (next section details) automatically and update the product design to product / process knowledge database, knowledge of self-learning.

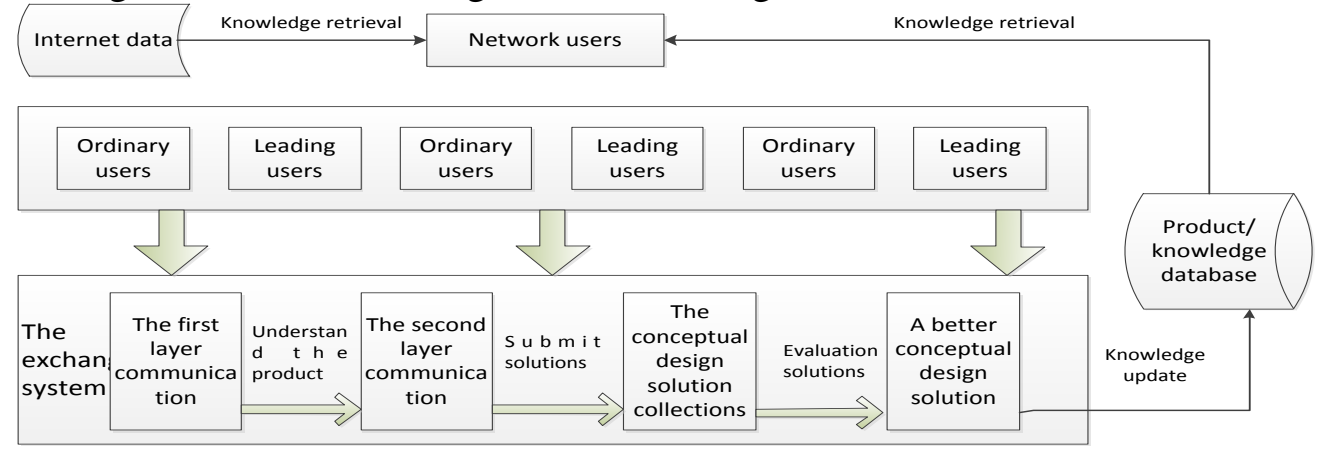

Fig.1 Structure of AC system

\subsection{System core function}

(1)Two layer communication of network users

Due to the presence of some utopian demand [8] of some users, these users will proposed some ideas and requirements completely according to their individual requirements. These requirements may be impractical and unmeaning. For this reason, the author designs a two layer exchange, the first layer exchange area let users understand and to be familiar with a product, after the first layer exchange and if we find the product concept belongs to the fantasy demand directly, we eliminate it, otherwise, into the second layer exchange and submit the concept product design, follow-up assessment at the same time, get a better conceptual design solution.

(2)Web browsing

Web browsing is a function that a user can watch the working interface of another online user. Introducing its main purpose is to facilitate a user to another user online demo, mainly refers to a product solution design CAD drawings demonstration, or two online users chat for the product structure, function, size and other details. Visible, network users can through web browsing capabilities more in-depth, more fully the concept of the product details of the exchange, and not just from the Internet, product / process knowledge database retrieval.

(3)Modeling and selection of the optimal product solution

Each product solution design is uniquely identifies the ID, design content, support, oppose, submitted to the time, product design set Product Solutions said formula (1), as shown in

ProductSolutions $=\{$ ps1, ps2...psI... $\}$ 


$$
\text { psI }=\{\text { idI, contentI, supportI, opposeI, timeI }\}
$$

So that the support accounted for alpha, against the proportion of the number of beta. 1 is greater than or equal to alpha $>$ beta is greater than or equal to 0 and alpha + beta $=1$ and the proportion of the number of support. The formula for the number of $M$ and $M$ is shown in the formula (3)

$$
\mathrm{N}
$$

$$
\mathrm{M}=\underset{1}{\mathrm{MAX}}\{\text { suppor } \mathbb{\mathrm { I }} * \alpha+\text { opposeI } * \beta\}
$$

Among, $\mathrm{M}$ is the maximum value of the supprotI* alpha +opposeI* beta from 1 to $\mathrm{N}$ product solutions.

In the calculation of the optimal product design, first, from the Product Solutions collection to remove the time distance is now more than one year of the solution, in order to pursue the effectiveness. Second, from the results of the calculation of $M$ to find the maximum value of the corresponding ID, then the ID corresponding solution design for the better product solution design.

(4)Knowledge learning function

Knowledge learning function make the better product conceptual design solution can be updated in real time to product / process knowledge database, Knowledge self-learning function can realize the cycle of knowledge utilization, obtain the excellent knowledge, and provide subsequent knowledge retrieval for the network users, so that provides a wealth of useful for the high quality of the knowledge.

\section{The System Implementation And Results Analysis}

Mainly to add product solution, A better product solution, Vote for the user's product design solution, Remote browsing function, four page to achieve, respectively, the corresponding figure 2, figure 3 and Figure 4 and Figure 5. System in the development process of reference [9, 10].

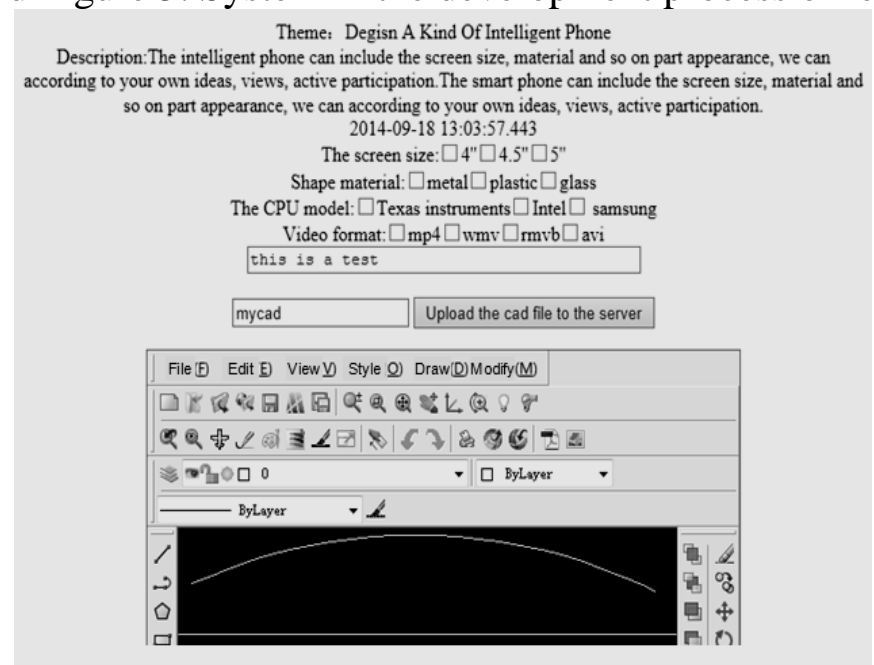

Fig.2 Add product solution

Figure 2 for the user can through the check box assembly of the product structure, can have a detailed description of their own ideas and detailed design in the text area, also can through the CAD software embedded in a web page rendering graph of the function and structure of the products to be submitted. 


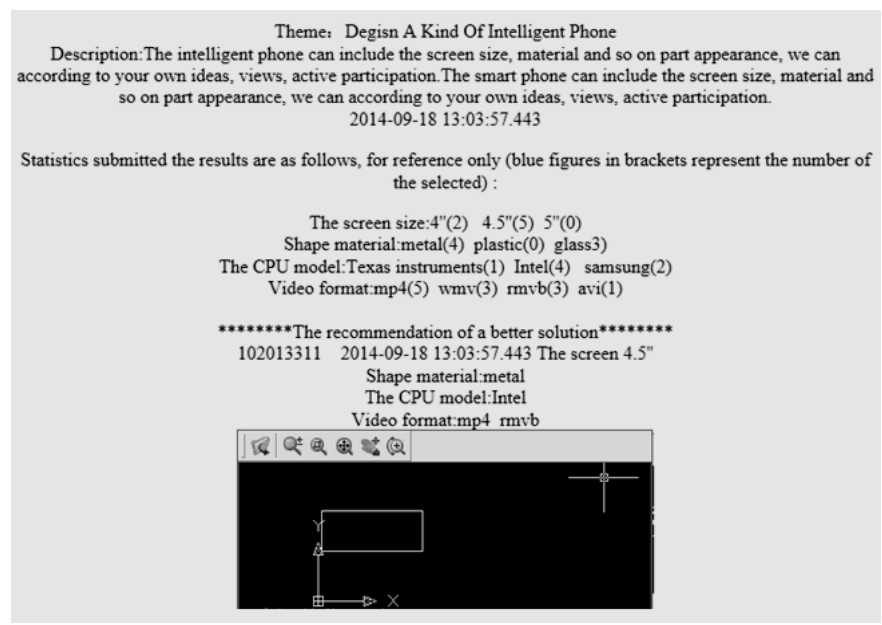

Fig.3 A better product solution

Figure 3 is more excellent product design and display results, consists of two parts, one part is that the number in the brackets are general statistics from all users' solutions for product structure properties and components; the other part is a better product design solution according to the Modeling and selection of the optimal product solution.

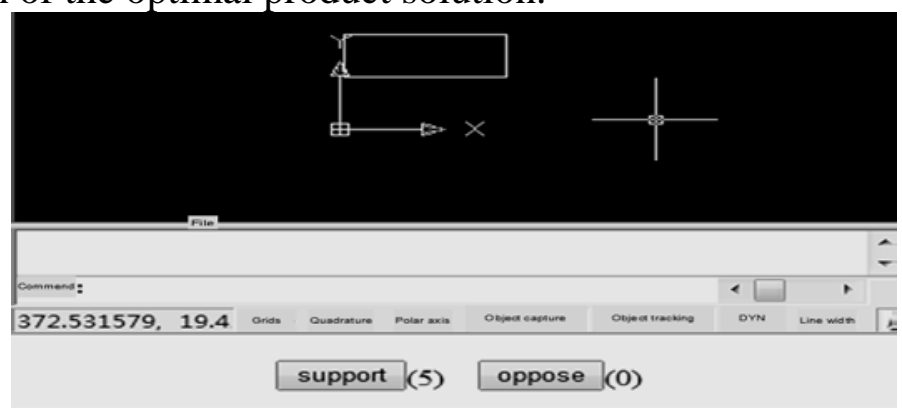

Fig.4 Vote for the user's product design solution

Figure 4 for the user can vote for the solutions has been submitted. You can choose one from the "support" button and "against" button or can’t express your view. But a user can only vote once for a solution.

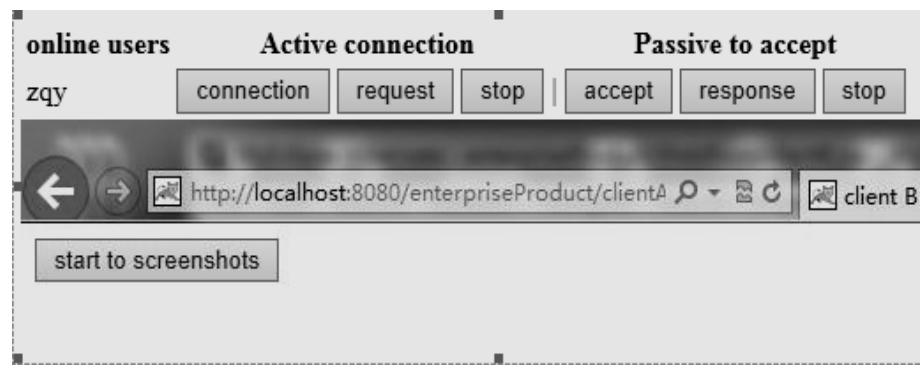

Fig.5 Remote browsing function

Figure 5 for the user's Web site online remote viewing function. Users can log on to online users and online users through real-time online interactive.

\section{Summary}

The role of product conceptual design for product innovation is self-evident, but the study of product conceptual design is mainly based on theory of TRIZ ,in order to repair, improve the above four problems combined with the research of the network community, the system is presented. From the system to achieve the effect, four problems have been improved to a certain extent. Although the problem is corrected, but the solutions' quality remains to be improvement, product conceptual design solution evaluation and choose optimization. These are the following further research content, also expect this paper can for product conceptual design research direction to provide a reference. 


\section{Acknowledgemnts}

This work has been supported by National Natural Science Foundation of China:51165004 and also supported by the project of scientific research and technological development plan in the Guangxi Zhuang Autonomous Region China in 2015: development of a kind of software system for open innovation conceptual design on products oriented to micro and small and medium sized enterprises.

\section{References}

[1]Zhang Jianhui,Tan Runhua,Zhang Peng etc. Computer aided design concept innovation-driven product generation process model innovative ideas[J]. Computer Integrated Manufacturing Systems. Vol.19(2013)No.2,p. 282-292.

[2]Li Pingping. Research and implementation of key technologies in product innovation design system[D](master, Shaanxi University of Science and Technology, 2013).p.13.

[3]Jiang Lili,Li Yan,Li Wenqiang. The expression model of product conceptual design based on multi angle of view[J]. Computer Integrated Manufacturing Systems. Vol.20(2014)No.5,p. 990-1002.

[4]Dong Zejia,Xu Zhengliang Research on enterprise information behavior based on network community [J]. Information theory and Practice. Vol.36(2013)No.11,p. 86-89.

[5]Hu Zhuo. Research on the process of product conceptual design based on TRIZ[D](master, Huazhong University of Science and Technology, 2011).p.18.

[6]He Chaobo,Tang Yong,Mai Huiqiang etc. Online social networks Summary [J]. Journal of Wuhan University (SCIENCE EDITION). Vol.60(2014)No.3,p.190-200.

[7]Zhang Shu. Research on the influence factors of user participation innovation in network innovation community[D](master, Northeastern University, 2012).p.25.

[8]Li Juntao,Jiang Lijun. Application of cloud innovation in industrial product innovation design[J]. Mechanical design and manufacturing. (2013)No.5,p.60-66.

[9]Li Han. Application and research of social network development framework based on Web2.0 [D](master, Shanghai Jiao Tong University, 2010).p.20.

[10]Fan Xiangyang:Research and implementation of enterprise social computing platform based on Web2.0[D](master, Shanghai Jiao Tong University, 2008). p.15. 